\title{
Nonrelativistic Open String Model - Magnetic Monopole Mass and Lifetime Values
}

\author{
Joseph Bevelacqua
}

Funding: The author(s) received no specific funding for this work.

Potential competing interests: The author(s) declared that no potential competing interests exist.

\begin{abstract}
Candidate magnetic monopole string parameters are investigated using a nonrelativistic open string model with fixed endpoints. String parameters and lifetime values are derived as a function of the magnetic monopole mass. A wide variation in string parameter and lifetime values is predicted for the various monopole mass values utilized in this paper. The monopole mass lifetime values exceed $10^{22} \mathrm{y}$ for the 10 to $10^{18} \mathrm{MeV} / \mathrm{c}^{2}$ mass range considered in this paper.
\end{abstract}

\subsection{Introduction}

String theory is an elegant mathematical formulation ${ }^{1-7}$ that has yet to be experimentally verified. Specific particle parameter values and associated decay modes are uncertain and have been qualitatively discussed $^{8-29}$. These uncertainties are exemplified by estimates of the monopole mass and lifetime values $^{25}$. This paper applies the nonrelativistic open string model proposed in Refs. 28 and 29 to calculate a range of magnetic monopole string parameter and lifetime values as a function of assumed monopole mass values. Since the magnetic monopole mass values are uncertain ${ }^{25}$, a wide range of values, encompassing 10 to $10^{18} \mathrm{MeV} / \mathrm{c}^{2}$, is utilized in this paper ${ }^{25}$. This range of mass values is based on a variety of assumed monopole origins ${ }^{25}$.

The magnitude of the monopole mass and associated lifetime values has implications for both particle physics and cosmology. Magnetic monopoles are predicted by a number of theories including string theory and various Grand Unification Theories. The discovery of magnetic monopoles would provide an importance benchmark for advancing various theories as well as the development or more comprehensive approaches including a better quantification of realistic Grand Unification Theories as well as the possible development of a Theory of Everything. In addition, monopole detection would open new research avenues in both particle physics and cosmology.

Using Refs. 25 and 27-29 as a guide, this paper defines a model to calculate the monopole lifetime and associated string parameters as a function of magnetic monopole mass using the nonrelativistic open string model with fixed endpoints 28,29 . By constraining the model to reproduce a selected monopole mass, a set of parameters that provide an initial representation for the monopole string and associated lifetime 
are derived.

Determination of these string parameters and lifetime values is fraught with obvious uncertainty. The present approach provides string parameters that establish an initial, but not definitive, set as the basis to explore in future work. As noted in Refs. 28 and 29, subsequent work will include a model string incorporating charge, electric and magnetic fields, multiple interacting strings including loops, various boundary conditions, interaction types, gauge theories, and symmetry conditions. The deviation in string parameters from the base case established in this paper will illuminate the dependence of the various parameters on specific string properties.

\subsection{Nonrelativistic Open String Model Overview}

The model proposed in this paper assumes the production of cosmic strings following the big bang or during a big bang/crunch cycle of cosmic events. In this paper, it is assumed that particles result from the emission of the vibrational energy of the string. The fields associated with these particles can be derived from a number of symmetry classes. A simple example would be an Abelian-Higgs theory with a complex scalar field and a $U(1)$ gauge field ${ }^{27-29}$. This class of fields is shown by Matsunami et al. ${ }^{27}$ to produce a string with a lifetime, defined in Section 6.0 that is proportional to the square of the string length.

Following the Abelian-Higgs field theory with a $\mathrm{U}(1)$ gauge approach, the decay of strings into requisite particles occurs episodically with an associated energy loss. This energy loss is associated with the magnetic monopole mass

In Ref. 28, a representative sample of string parameters for a set of baryons, leptons, and mesons was determined. This determination was based on specific mass and lifetime values for the set of selected particles that included the proton, neutron, and lambda baryons; electron, muon, and tau leptons; and charged pions and charged B mesons ${ }^{28}$. In Ref. 29, neutrino string parameters and lifetime values were determined in a similar manner.

Since the magnetic monopole mass and lifetime values are uncertain ${ }^{25}$, these circumstances require a somewhat different approach than utilized in Ref. 28. The approach that is utilized is based on the approach of Ref. 29. Given these uncertainties, magnetic monopole masses are assumed to vary between 10 and $10^{18} \mathrm{MeV} / \mathrm{c}^{2}$ where this mass range is suggested in Ref. 25. For each assumed mass, string parameter and lifetime values are derived from the best three fits to the particle mass value. These parameter values and lifetimes are summarized in Table $1-5$.

\subsection{Model Parameter Specification}

The string model utilized in this paper is limited to nonrelativistic velocities. The energy of the string available for monopole decay is based on its total vibrational energy (kinetic plus potential energy). In this paper, assumed monopole mass values are utilized to calculate the associated lifetime and string parameter values.

Key model parameters include the string density, which is related to the tension, and the length, amplitude, and velocity. Bounds on the string tension (S), derived from pulsar timing measurements $22-24$, 27 , are based on the gravitational wave background produced by decaying cosmic string loops. This bound, 
GS $\leq 10^{-11}$, is based on Newton's gravitational constant $(G)$ and is derived from simulations that ignore the field composition of the string. This would correspond to a string mass density of about $1.4 \times 10^{17} \mathrm{~kg} / \mathrm{m}$. As a matter of comparison, a density of $1.4 \times 10^{27} \mathrm{~kg} / \mathrm{m}$ is derived from the Planck energy divided by the Planck length. Ref. 20 suggests that a string density of $10^{21} \mathrm{~kg} / \mathrm{m}$ is an appropriate string density. These results imply that a range of density values are possible. Accordingly, the string density is permitted to vary over a range of values.

Matsunami et al. ${ }^{27}$ suggest that particle radiation is associated with a string length that is $<10^{-19} \mathrm{~m}$. Longer-lived particles that do not decay or that have extended lifetimes (e.g., protons and electrons) would be expected to have significantly longer string lengths. This assertion was also noted in Refs. 28 and 29. In addition, cosmological strings are expected to be mildly relativistic ${ }^{27}$. Ref. 27 utilizes values of 0.33 c and $0.6 \mathrm{c}$ in their calculations. The model proposed in this paper 28,29 uses a nonrelativistic approach and limits the string velocity to values less than used in Ref. 27 (i.e., $\beta \leq 0.05$ ).

These parameter values will be used as a guide and not a specific limitation in this paper. Reasonable variations will be considered in subsequent discussion. In particular, the density is permitted to vary between $10^{7}$ and $1.4 \times 10^{27} \mathrm{~kg} / \mathrm{m}$. The string length is permitted to vary within the $10^{-21}$ to $10^{46} \mathrm{~m}$. As noted above, the string velocity is assumed to be nonrelativistic. Amplitude values are restricted to be less than the string length.

\subsection{Base Case String Model}

Cosmic strings have extremely large masses that greatly exceed the values considered in this paper. The particle masses are assumed to be generated by the kinetic and potential energies of the vibrating string. The resulting particle mass does not depend on the total inclusive string mass. In this paper, the inherent string mass is treated as a renormalized vacuum or zero point energy with particles associated with the vibrational energy of the string.

As a base case, a one-dimensional string of finite length and fixed endpoints is assumed. The model details are provided in Refs. 28 and 29 and only salient features will be addressed in this paper.

\subsection{Magnetic Monopole Mass}

Assuming a uniform energy density over the string length, the energy ( $E$ ) of a particle corresponding to the string vibrational energy density 28,29 with total length $L$ is

$$
E=\frac{1}{2} \mu A^{2} \omega^{2} L(1)
$$

where $\mu$ is the string mass per unit length, $A$ is the amplitude, and $\omega$ is the angular frequency.

An application of Eq. 1 permits an estimate of the magnetic monopole's rest mass energy $(\varepsilon)$. As noted in Refs. 28 and 29, Eq. 1 can be written as

$$
E=2 \pi^{2} \mu A^{2 \frac{v^{2}}{\lambda^{2}}} L={ }^{\frac{\pi^{2}}{2}} \mu A^{2 \frac{v^{2}}{L}} \approx \epsilon(2)
$$


where $\lambda=2 \mathrm{~L}$ based on a first harmonic assumption ${ }^{28,29}$, and $\mathrm{v}$ is the string velocity.

\subsection{Magnetic Monopole Lifetime}

Matsunami et al. ${ }^{27}$ provide a relationship for the string lifetime $(\tau)$

$$
\tau \approx \frac{S L^{2}}{\xi \epsilon C}=\frac{v^{2} \mu L^{2}}{\xi \epsilon C}
$$

where $\xi$ is the number of episodes per period, and $\varepsilon$ is the average energy lost per unit time which the model assumes to be the magnetic monopole rest mass energy. The string described in Section 4 is used as the basis for estimating the magnetic monopole lifetime.

\subsection{Model Assumptions and Limitations}

The magnetic monopole lifetime and associated string parameters are derived by assuming the following:

1. The model, defined in Sections 2 - 4, specifies the string parameters that characterize the monopole.

2. One episode per period is assumed which is consistent with the fundamental mode assumption of Section 5.

3. The average energy lost per unit time (e.g., over a period) is the string kinetic plus potential energy. Since the string is nonrelativistic, this is assumed to be the monopols's rest mass. The magnetic monopole lifetime is derived from the rest mass energy of the particle $(\varepsilon)$ and is defined by Eqs. 2 and 3.

4 Only the string kinetic plus potential energy contributes to the monopole mass. The inherent string mass $\left(\bar{\rho}_{L}\right)$ is essentially a renormalizable constant (i.e., it is the vacuum or zero point energy), because the magnetic monopole energy is much smaller than this inherent mass.

5. The specific magnetic monopole decay modes and their associated decay products are not specified or considered.

\subsection{Results and Discussion}

The model results provide specific magnetic monopole string parameter and lifetime values as a function of mass. Model results suggest that long-lived magnetic monopole lifetime values are obtained for a wide range of string parameters. The string parameters (i.e., density, length, amplitude, and velocity) supporting these lifetime values are addressed, and their variation with monopole mass are discussed in subsequent commentary. Tables 1, 2, 3, 4, and 5 summarize, as a function of magnetic monopole mass, the monopole string density, length, amplitude, beta value, and lifetime values, respectively. The three best fits to the assumed monopole mass are provided in these tables.

Given the nature of the proposed calculations and associated uncertainties, a preliminary goal of fitting the particle masses and lifetimes to within $1 \%$ of their assumed values was set. This appears to be a reasonable criterion for the initial calculations.

In Tables 1 - 5, the notation $\mathrm{H}$ (high), M (medium), and L (low) is used to label the columns of the three best parameter fits to the assumed magnetic monopole mass value. The parameter set yielding the largest 
lifetime for each string mass is listed under the $H$ column. The $L(M)$ columns record the lowest (middle) lifetime for each of the assumed magnetic monopole mass values.

\subsection{Magnetic Monopole Masses}

The magnetic monopole masses summarized in Tables $1-5$ are limited to values between 10 and $10^{18}$ $\mathrm{MeV} / \mathrm{c}^{2} 25$. The string parameters and lifetime values are calculated as a function of these assumed magnetic monopole mass values. The monopole mass values were fit to within $0.1 \%$ for all masses considered in Tables 1 - 5 .

Given the simplistic nonrelativistic, uncharged, fixed endpoint open string model, the mass results are encouraging. However, the model parameter assumptions and associated parameter ranges are still lacking in experimental verification.

\subsection{String Density}

As noted in Table 1, there is significant variation in the string density as a function of magnetic monopole mass for the $\mathrm{L}, \mathrm{M}$, and $\mathrm{H}$ Cases. In particular, the string density values reside within the range of $10^{10}-10^{27} \mathrm{~kg} / \mathrm{m}$. In view of this variation, definitive conclusions regarding the string density are not possible. Therefore, a more global analysis must be utilized.

\section{Table 1}

Magnetic Monopole String Density $(\mathrm{kg} / \mathrm{m})^{a}$

\begin{tabular}{|l|l|l|l}
\hline Monopole Mass (MeV) & Case L & Case M & Case H \\
\hline $10^{1}$ & $6.05 \times 10^{26}$ & $1.29 \times 10^{24}$ & $5.57 \times 10^{23}$ \\
\hline $10^{2}$ & $4.97 \times 10^{12}$ & $3.52 \times 10^{13}$ & $1.98 \times 10^{26}$ \\
\hline $10^{3}$ & $2.84 \times 10^{12}$ & $5.13 \times 10^{20}$ & $3.58 \times 10^{17}$ \\
\hline $10^{4}$ & $1.09 \times 10^{18}$ & $3.76 \times 10^{12}$ & $1.19 \times 10^{21}$ \\
\hline $10^{5}$ & $3.24 \times 10^{10}$ & $1.25 \times 10^{16}$ & $1.17 \times 10^{17}$ \\
\hline $10^{6}$ & $3.29 \times 10^{14}$ & $8.83 \times 10^{16}$ & $9.13 \times 10^{24}$ \\
\hline $10^{7}$ & $1.91 \times 10^{18}$ & $3.19 \times 10^{23}$ & $2.53 \times 10^{18}$ \\
\hline $10^{8}$ & $1.09 \times 10^{18}$ & $3.40 \times 10^{22}$ & $1.55 \times 10^{17}$ \\
\hline $10^{9}$ & $1.91 \times 10^{18}$ & $4.50 \times 10^{22}$ & $1.79 \times 10^{19}$ \\
\hline $10^{10}$ & $2.08 \times 10^{21}$ & $3.63 \times 10^{21}$ & $1.50 \times 10^{26}$ \\
\hline $10^{11}$ & $9.91 \times 10^{10}$ & $1.42 \times 10^{14}$ & $8.00 \times 10^{26}$ \\
\hline $10^{12}$ & $1.19 \times 10^{21}$ & $8.69 \times 10^{12}$ & $6.36 \times 10^{21}$ \\
\hline $10^{13}$ & $8.83 \times 10^{16}$ & $5.95 \times 10^{22}$ & $8.00 \times 10^{26}$ \\
\hline $10^{14}$ & $2.15 \times 10^{12}$ & $1.62 \times 10^{12}$ & $4.65 \times 10^{13}$ \\
\hline $10^{15}$ & $7.25 \times 10^{19}$ & $1.29 \times 10^{24}$ & $8.00 \times 10^{26}$ \\
\hline $10^{16}$ & $9.28 \times 10^{11}$ & $1.40 \times 10^{10}$ & $5.22 \times 10^{24}$ \\
\hline $10^{17}$ & $7.74 \times 10^{18}$ & $7.74 \times 10^{18}$ & $8.00 \times 10^{26}$ \\
\hline $10^{18}$ & $1.52 \times 10^{13}$ & $1.95 \times 10^{22}$ & $6.46 \times 10^{25}$ \\
\hline & & & \\
\hline
\end{tabular}

${ }^{a}$ Cases $\mathrm{L}($ low), $\mathrm{M}$ (Medium), and $\mathrm{H}$ (high) are based on the relative lifetime values of Table 5. 
To facilitate a global analysis, an averaged logarithmic string parameter (ALSP) $\Omega(E)$ is defined by the relationship:

$$
\log _{10} \Omega(E)=\frac{\log _{10} \Omega_{L}(E)+\log _{10} \Omega_{M}(E)+\log _{10} \Omega_{H}(E)}{3}
$$

where the averaged logarithmic string parameters are ALS $\mu$ for the string density, ALSL for the string length, ALSA for the string amplitude, and ALAt for the string lifetime. The averaged string velocity (AS $\beta$ ) is addressed in subsequent discussion.

The ALS $\mu$ for the string density is plotted as a function of magnetic monopole mass in Fig. 1. As expected, the ALS $\mu$ (Fig. 1 dashed curve derived from the Table 1 data) still exhibits considerable variation, but it is less severe than the individual Case $L, M$, and $H$ variations.

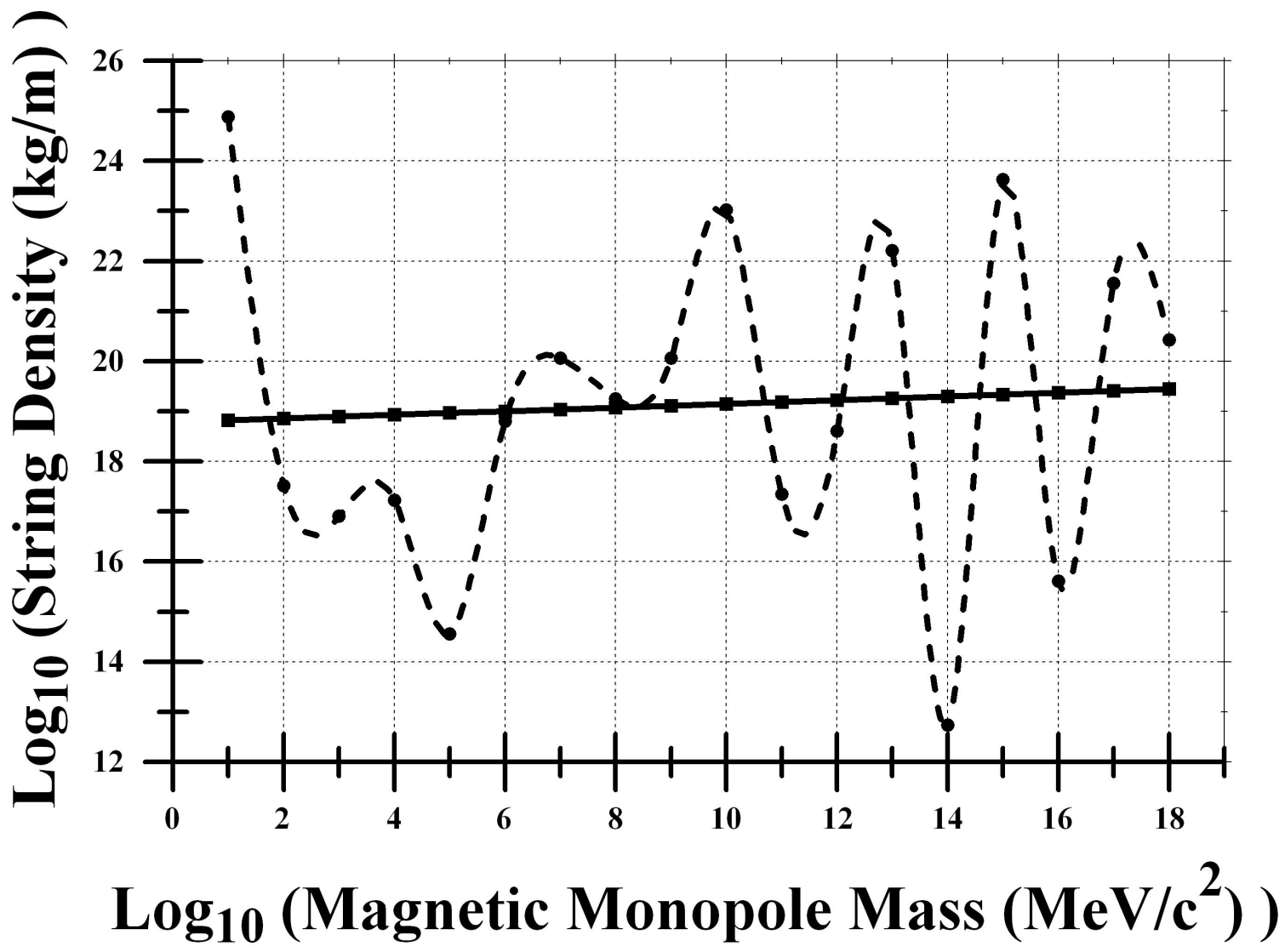

Figure 1 Magnetic monopole string density as a function of magnetic monopole mass

The solid curve in Table 1 represents a linear fit to the ALS $\mu$ values defined by the relationship:

$$
\mu(E)=a \log _{10} \mu_{A L S \mu}(E)+b(5)
$$


where $a=0.037043636 \mathrm{~kg} / \mathrm{m}$ and $b=18.78218456 \mathrm{~kg} / \mathrm{m}$. The linear fit suggests an averaged string density on the order of $10^{19} \mathrm{~kg} / \mathrm{m}$ for monopole masses in the range of $10-1.0 \times 10^{18} \mathrm{MeV} / \mathrm{c}^{2}$.

The monopole sting density is higher than noted in Ref. 28 for unstable baryons (neutrons and lambdas), leptons (muons and taus), and mesons (charged pions and charged B mesons). However, the electron and proton string density range of values encompasses the range of magnetic monopole values of $10^{10}-10^{27} \mathrm{~kg} / \mathrm{m}^{29}$.

Baryon densities derived in Ref. 28 were $10^{12}-10^{18} \mathrm{~kg} / \mathrm{m}$ for neutrons, $10^{10}-10^{27}$ for protons, and about $10^{12} \mathrm{~kg} / \mathrm{m}$ for lambdas. Lepton string densities also tend to be lower than the corresponding magnetic monopole values with values of $10^{11}-10^{21} \mathrm{~kg} / \mathrm{m}, 10^{12}-10^{16} \mathrm{~kg} / \mathrm{m}$, and $10^{11}-10^{12} \mathrm{~kg} / \mathrm{m}$ for electron, muon, and tau leptons, respectively ${ }^{28}$. Meson string densities for charged pions $\left(10^{11}-10^{14}\right.$ $\mathrm{kg} / \mathrm{m})$ and charged $B$ mesons $\left(\approx 10^{11} \mathrm{~kg} / \mathrm{m}\right)$ also exhibit a lower value than the magnetic monopole string density.

The results of Ref. 28 suggest that higher string densities are exhibited for long-lived particles. Although the monopole lifetime is not well established ${ }^{25}$, the string density results suggest the magnetic monopole is also a long-lived particle with a lifetime range, noted in Table 5, which is similar to that of the proton and electron.

\subsection{String Length}

Following Ref. 27, the string length associated with the decay of unstable particles should be $<10^{-19} \mathrm{~m}$. As noted in previous discussion, this value provides an indication of an expected unstable particle string length, and the results of other open string nonrelativistic models may differ.

The monopole string length values summarized in Table 2 vary over a range of $10^{6}-10^{17} \mathrm{~m}$. These string length values are much larger than noted for unstable particles 27,28 .

For baryons, the neutron and lambda string lengths are in the range of $10^{-15}$ to $10^{-12} \mathrm{~m}$ and $\approx 10^{-19} \mathrm{~m}$, respectively ${ }^{28}$. A similar range of string values is found for short-lived leptons. The muon and tau string lengths are in the range of $10^{-19}$ to $10^{-17} \mathrm{~m}$ and $\approx 10^{-19} \mathrm{~m}$, respectively. The meson values are $10^{-19}$ to $10^{-}$ $17 \mathrm{~m}$ and $\approx 10^{-19} \mathrm{~m}$ for the charged pion and $\mathrm{B}$ meson, respectively.

For long-lived particles, string lengths have an increased value. Proton and electron string lengths are in the range of $10^{6}-10^{11} \mathrm{~m}$ and $10^{4}-10^{14} \mathrm{~m}$, respectively ${ }^{28}$. Eq. 3 suggests that the increased proton and electron lifetime values should correspond with string lengths that are much longer than those values encountered in unstable baryons, leptons, and mesons ${ }^{28}$. The results summarized in Table 2 further suggest a long-lived magnetic monopole. 
Table 2

Magnetic Monopole String Length $(\mathrm{m})^{\text {a }}$

\begin{tabular}{|l|l|l|l|}
\hline Monopole Mass $(\mathrm{MeV})$ & Case $\mathrm{L}$ & Case M & Case H \\
\hline $10^{1}$ & $3.42 \times 10^{8}$ & $3.71 \times 10^{13}$ & $8.57 \times 10^{15}$ \\
\hline $10^{2}$ & $8.02 \times 10^{10}$ & $1.46 \times 10^{12}$ & $1.76 \times 10^{12}$ \\
\hline $10^{3}$ & $1.80 \times 10^{14}$ & $1.09 \times 10^{10}$ & $1.86 \times 10^{12}$ \\
\hline $10^{4}$ & $7.50 \times 10^{7}$ & $1.46 \times 10^{11}$ & $1.75 \times 10^{9}$ \\
\hline $10^{5}$ & $8.65 \times 10^{9}$ & $1.18 \times 10^{12}$ & $1.15 \times 10^{13}$ \\
\hline $10^{6}$ & $1.65 \times 10^{6}$ & $3.34 \times 10^{11}$ & $1.38 \times 10^{12}$ \\
\hline $10^{7}$ & $9.50 \times 10^{11}$ & $2.16 \times 10^{11}$ & $3.99 \times 10^{16}$ \\
\hline $10^{8}$ & $2.62 \times 10^{9}$ & $2.10 \times 10^{7}$ & $5.53 \times 10^{10}$ \\
\hline $10^{9}$ & $9.50 \times 10^{11}$ & $4.51 \times 10^{10}$ & $5.00 \times 10^{12}$ \\
\hline $10^{10}$ & $9.90 \times 10^{9}$ & $5.60 \times 10^{10}$ & $1.65 \times 10^{9}$ \\
\hline $10^{11}$ & $6.89 \times 10^{15}$ & $5.92 \times 10^{16}$ & $9.70 \times 10^{12}$ \\
\hline $10^{12}$ & $1.53 \times 10^{7}$ & $1.52 \times 10^{12}$ & $5.73 \times 10^{11}$ \\
\hline $10^{13}$ & $2.93 \times 10^{9}$ & $2.77 \times 10^{12}$ & $9.70 \times 10^{12}$ \\
\hline $10^{14}$ & $1.93 \times 10^{6}$ & $2.22 \times 10^{8}$ & $1.54 \times 10^{13}$ \\
\hline $10^{15}$ & $2.91 \times 10^{14}$ & $2.05 \times 10^{13}$ & $9.70 \times 10^{12}$ \\
\hline $10^{16}$ & $7.89 \times 10^{9}$ & $6.79 \times 10^{14}$ & $2.44 \times 10^{10}$ \\
\hline $10^{17}$ & $2.22 \times 10^{8}$ & $5.58 \times 10^{13}$ & $7.96 \times 10^{9}$ \\
\hline $10^{18}$ & $7.26 \times 10^{9}$ & $6.77 \times 10^{15}$ & $1.86 \times 10^{15}$ \\
\hline
\end{tabular}

${ }^{a}$ Cases $\mathrm{L}($ low), $\mathrm{M}$ (Medium), and $\mathrm{H}$ (high) are based on the relative lifetime values of Table 5 .

The magnetic monopole string length results are further summarized in Fig. 2. In Fig. 2, the dashed curve represents the ALSL values derived from Table 2. The solid curve in Fig. 2 represents a linear fit to the ALSL values:

$$
L(E)=\operatorname{alog}_{10} L_{A L S L}(E)+b(6)
$$

where $\mathrm{a}=0.032089525 \mathrm{~m}$ and $\mathrm{b}=11.25266080 \mathrm{~m}$. The linear fit of Eq. 6 suggests an averaged string length of about $10^{11}-10^{12} \mathrm{~m}$ for monopole masses between 10 and $10^{18} \mathrm{MeV} / \mathrm{c}^{2}$. 


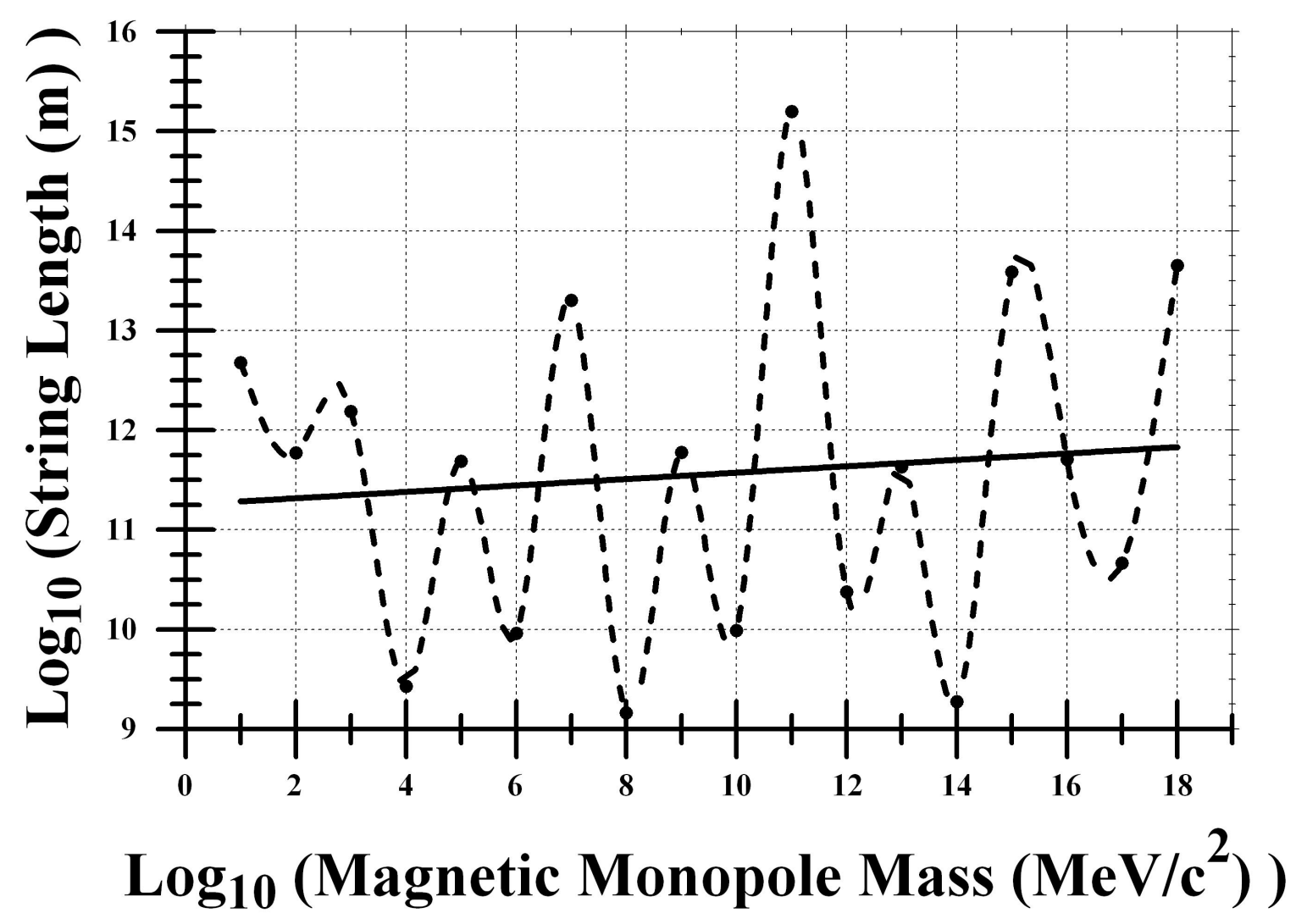

Figure 2 Magnetic monopole string length as a function of magnetic monopole mass

\subsection{String Amplitude}

The monopole string amplitude summarized in Table 3 has a range between $10^{-22}$ and $10^{-4} \mathrm{~m}$. As noted with the other string parameters, there is considerable variability in the amplitude values. This variability is reduced using the ALSA values. 
Table 3

Magnetic Monopole String Amplitude $(m)^{a}$

Monopole Mass (MeV)

Case L

Case M

Case $\mathrm{H}$

$10^{1}$

$5.83 \times 10^{-23}$

$3.42 \times 10^{-19}$

$5.03 \times 10^{-18}$

$10^{2}$

$5.55 \times 10^{-14}$

$7.29 \times 10^{-14}$

$2.26 \times 10^{-19}$

$10^{3}$

$7.45 \times 10^{-12}$

$2.78 \times 10^{-18}$

$4.81 \times 10^{-15}$

$10^{4}$

$1.40 \times 10^{-17}$

$2.60 \times 10^{-13}$

$8.08 \times 10^{-18}$

$10^{5}$

$5.03 \times 10^{-12}$

$6.66 \times 10^{-14}$

$3.83 \times 10^{-14}$

$10^{6}$

$3.27 \times 10^{-15}$

$3.57 \times 10^{-14}$

$8.65 \times 10^{-18}$

$10^{7}$

$3.15 \times 10^{-13}$

$3.53 \times 10^{-17}$

$7.56 \times 10^{-12}$

$10^{8}$

$1.42 \times 10^{-14}$

$5.79 \times 10^{-18}$

$1.09 \times 10^{-13}$

$10^{9}$

$3.15 \times 10^{-13}$

$3.04 \times 10^{-15}$

$1.06 \times 10^{-12}$

$10^{10}$

$4.30 \times 10^{-15}$

$7.20 \times 10^{-15}$

$1.16 \times 10^{-17}$

$10^{11}$

$4.45 \times 10^{-6}$

$7.82 \times 10^{-8}$

$8.36 \times 10^{-14}$

$10^{12}$

$2.00 \times 10^{-15}$

$1.60 \times 10^{-8}$

$2.71 \times 10^{-13}$

$10^{13}$

$1.02 \times 10^{-11}$

$8.93 \times 10^{-13}$

$8.36 \times 10^{-14}$

$10^{14}$

$1.46 \times 10^{-10}$

$5.30 \times 10^{-9}$

$1.26 \times 10^{-6}$

$10^{15}$

$10^{16}$

$2.38 \times 10^{-9}$

$2.21 \times 10^{-12}$

$8.36 \times 10^{-14}$

$4.61 \times 10^{-7}$

$3.55 \times 10^{-4}$

$1.66 \times 10^{-13}$

$10^{17}$

$6.78 \times 10^{-11}$

$2.34 \times 10^{-8}$

$2.33 \times 10^{-14}$

$10^{18}$

$1.01 \times 10^{-6}$

$1.48 \times 10^{-8}$

$7.96 \times 10^{-11}$

${ }^{a}$ Cases $\mathrm{L}($ low), $\mathrm{M}$ (Medium), and $\mathrm{H}$ (high) are based on the relative lifetime values of Table 5 .

Using Eq. 4, an ALSA value is calculated and is represented by the dashed curve in Fig. 3. The solid curve in Fig. 3 represents the linear fit to the ALSA values

$$
A(E)=\operatorname{alog}_{10} A_{A L S A}(E)+b(7)
$$

where $a=0.500573310 \mathrm{~m}$ and $\mathrm{b}=-17.27445508 \mathrm{~m}$. Over the magnetic monopole mass range of $10-$ $10^{18} \mathrm{MeV} / \mathrm{c}^{2}$, the string amplitude varies between $10^{-17}$ and $10^{-8} \mathrm{~m}$, respectively. This amplitude is significantly larger than the values for short-lived baryons, leptons, and mesons ${ }^{28}$. 


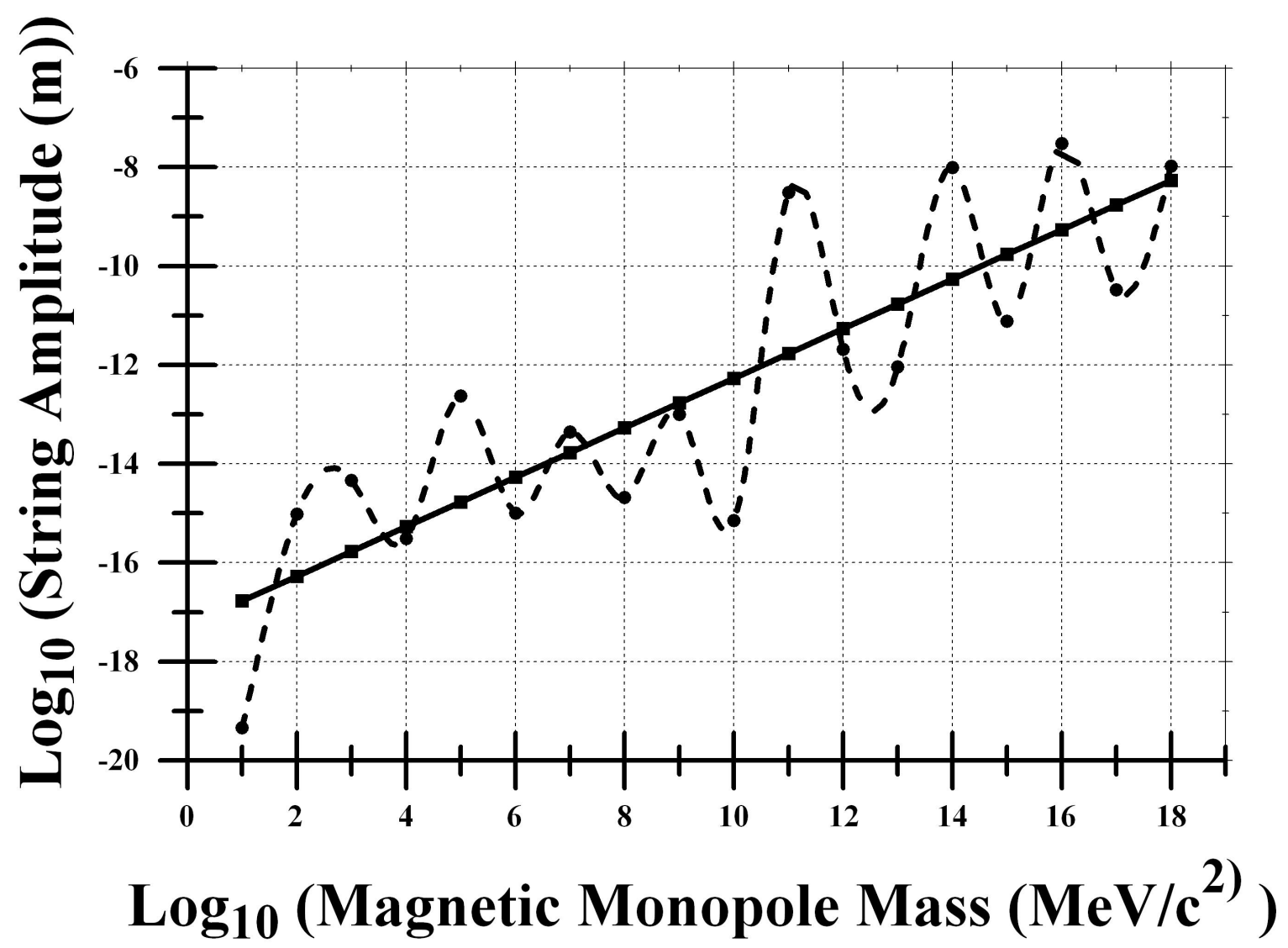

Figure 3 Magnetic monopole string amplitude as a function of magnetic monopole mass

The neutron amplitude is in the range of $10^{-29}$ to $10^{-25} \mathrm{~m}$, and the heavier lambda amplitude is $\approx 10^{-28}$ m. For short-lived leptons and mesons, larger amplitude values suggest a larger mass and shorter lifetime. The muon amplitude is in the range of $10^{-30}$ to $10^{-27} \mathrm{~m}$, and the heavier tau has an amplitude of $\approx 10^{-27} \mathrm{~m}$. Meson amplitudes follow a similar pattern, but the differences are not as large. The charged pion amplitude is in the range of $10^{-29}$ to $10^{-26} \mathrm{~m}$, and the heavier charged $B$ meson has a value of $\approx 10^{-27} \mathrm{~m}$.

The magnetic monopole amplitude is also larger in magnitude than the proton and electron values ${ }^{28}$. As noted in Reference 28, the proton and electron amplitude values are in the range of $10^{-20}-10^{-13} \mathrm{~m}$ and $10^{-19}-10^{-17} \mathrm{~m}$, respectively. These results suggest that long-lived particles have larger amplitudes than short-lived particles ${ }^{28}$. This result continues to suggest that the magnetic monopole has a long lifetime.

\subsection{String Velocity}

The string velocity is restricted to $\beta \leq 0.05$. In Reference 28 , the baryon, lepton, and meson results suggest that there is no general velocity relationship between values of $\beta$ and the particle mass or lifetime and associated string parameters. Similar results occur for the neutrino results ${ }^{29}$. There is also considerable scatter in the magnetic monopole string velocity values summarized in Table 4. 
Table 4

\begin{tabular}{|c|c|c|c|}
\hline Monopole Mass (MeV) & Case L & Case M & Case H \\
\hline $10^{1}$ & 0.0245 & 0.0298 & 0.0468 \\
\hline $10^{2}$ & 0.0138 & 0.0168 & 0.00250 \\
\hline $10^{3}$ & 0.0203 & 0.0315 & 0.00900 \\
\hline $10^{4}$ & 0.0355 & 0.0455 & 0.00900 \\
\hline $10^{5}$ & 0.0195 & 0.0278 & 0.0493 \\
\hline $10^{6}$ & 0.0130 & 0.0328 & 0.0270 \\
\hline $10^{7}$ & 0.00425 & 0.0443 & 0.0315 \\
\hline $10^{8}$ & 0.0208 & 0.0258 & 0.0330 \\
\hline $10^{9}$ & 0.0425 & 0.00625 & 0.00950 \\
\hline $10^{10}$ & 0.0305 & 0.0328 & 0.0173 \\
\hline $10^{11}$ & 0.0113 & 0.0495 & 0.000250 \\
\hline $10^{12}$ & 0.0340 & 0.0158 & 0.0210 \\
\hline $10^{13}$ & 0.0338 & 0.0145 & 0.00250 \\
\hline $10^{14}$ & 0.0390 & 0.0133 & 0.00275 \\
\hline $10^{15}$ & 0.0160 & 0.0343 & 0.0250 \\
\hline $10^{16}$ & 0.0120 & 0.0373 & 0.0248 \\
\hline $10^{17}$ & 0.0150 & 0.0218 & 0.0258 \\
\hline $10^{18}$ & 0.0130 & 0.0240 & 0.0405 \\
\hline
\end{tabular}

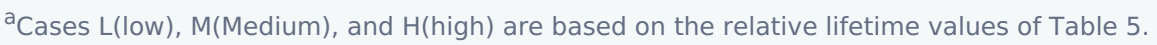

The $L, M$, and $H$ Case values were averaged to obtain the AS $\beta$ value:

$$
\beta_{A S \beta}(E)=\frac{\beta_{L}(E)+\beta_{M}(E)+\beta_{H}(E)}{3}
$$

where the $\beta_{A S \beta}(E)$ values were fit to the linear relationship

$$
\beta=a \beta_{A S \beta}(E)+b(9)
$$

with $\mathrm{a}=-0.00019091847$ and $\mathrm{b}=0.025519281$.

In Fig. 4, the dashed curve represents the $\beta_{A S \beta}(E)$ values, and the solid curve illustrates the linear fit values of Eq. 9. The averaged $\beta_{A S \beta}(E)$ values still exhibit considerable scatter, but the linear fit suggests the monopole velocity values lie in the range of about $0.022-0.025$. 


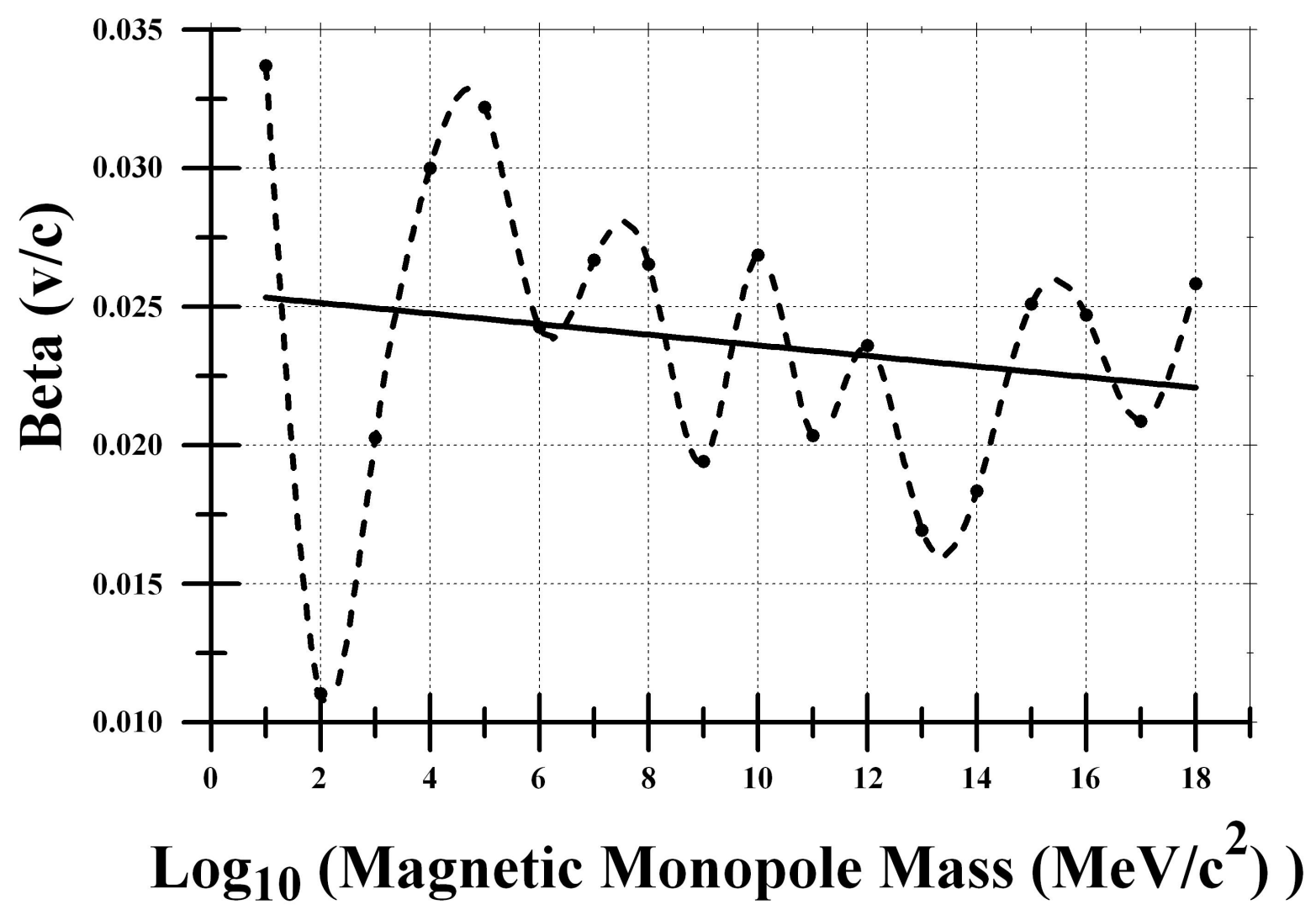

Figure 4 Magnetic monopole string velocity as a function of magnetic monopole mass

The Table 4 and Fig. 4 values are not clustered near the maximum $\beta$ value (i.e., 0.05) that suggests that the model is favoring a nonrelativistic solution. This conclusion is model dependent and must be verified with a more refined approach including electromagnetic fields and other symmetry assumptions that were noted previously.

\subsection{Particle Lifetime}

Following Eq. 3 and the associated discussion, the particle lifetime values are strongly dependent on the string length, tension, and particle mass. The particle mass (Eq. 2) involves multiple parameters, but the lifetime (Eq. 3) only depends on a subset of these parameters.

The variation in lifetime values as a function of magnetic monopole mass is illustrated by an examination of Table 5. As summarized in Table 5, the monopole lifetime values vary significantly and range between about $10^{22}$ and $10^{66} \mathrm{y}$. In the spirit of the model assumptions and limitations, the results of Table 5 were fit to the functional form of Eq. 4. 
Table 5

Magnetic Monopole String Mean Lifetime $(y)^{a}$

Monopole Mass (MeV)

Case

$10^{1}$

$10^{2}$

$2.53 \times 10^{53}$

Case M

Case $\mathrm{H}$

$10^{3}$

$3.59 \times 10^{42}$

$9.33 \times 10^{60}$

$5.31 \times 10^{65}$

104

$2.23 \times 10^{48}$

$1.25 \times 10^{46}$

$2.28 \times 10^{57}$

$10^{4}$

$10^{5}$

$4.61 \times 10^{40}$

$3.61 \times 10^{48}$

$5.94 \times 10^{48}$

$5.47 \times 10^{35}$

$9.87 \times 10^{41}$

$1.74 \times 10^{45}$

$10^{6}$

$8.98 \times 10^{30}$

$8.00 \times 10^{45}$

$2.24 \times 10^{49}$

$10^{7}$

$1.85 \times 10^{44}$

$6.30 \times 10^{44}$

$7.57 \times 10^{53}$

$10^{8}$

$1.92 \times 10^{39}$

$1.73 \times 10^{50}$

$2.37 \times 10^{55}$

$10^{9}$

$1.85 \times 10^{44}$

$5.92 \times 10^{39}$

$3.05 \times 10^{41}$

$10^{10}$

$1.13 \times 10^{42}$

$2.12 \times 10^{44}$

$2.40 \times 10^{45}$

$10^{11}$

$10^{12}$

$10^{13}$

$3.54 \times 10^{41}$

$7.27 \times 10^{43}$

$7.19 \times 10^{44}$

$7.26 \times 10^{47}$

$2.80 \times 10^{48}$

$1.91 \times 10^{34}$

$2.97 \times 10^{35}$

$5.46 \times 10^{43}$

$5.14 \times 10^{33}$

$5.69 \times 10^{44}$

$2.80 \times 10^{48}$

$10^{14}$

$7.26 \times 10^{21}$

$8.37 \times 10^{24}$

$4.97 \times 10^{34}$

$10^{15}$

$10^{16}$

$9.34 \times 10^{43}$

$3.79 \times 10^{46}$

$2.80 \times 10^{48}$

$4.94 \times 10^{25}$

$5.32 \times 10^{34}$

$1.13 \times 10^{40}$

$10^{17}$

$5.11 \times 10^{28}$

$6.78 \times 10^{39}$

$2.00 \times 10^{40}$

$10^{18}$

$8.04 \times 10^{24}$

$3.05 \times 10^{46}$

$2.18 \times 10^{49}$

${ }^{a}$ Cases $\mathrm{L}$ (low), $\mathrm{M}$ (Medium), and $\mathrm{H}$ (high) are based on the relative lifetime values of this table.

The ALSt values are plotted in Fig. 5 (dashed curve) and exhibit considerable variation. In Fig. 5, the solid curve represents the linear fit to the ALST values

$$
\tau(E)=\operatorname{alog}_{10} \tau_{A L S \tau}(E)+b(10)
$$

where the parameters $a=-0.90480678$ y and $b=51.63738806$ y. The linear fit provides a more stable set of lifetime values, but there is still a significant variation with mass. 


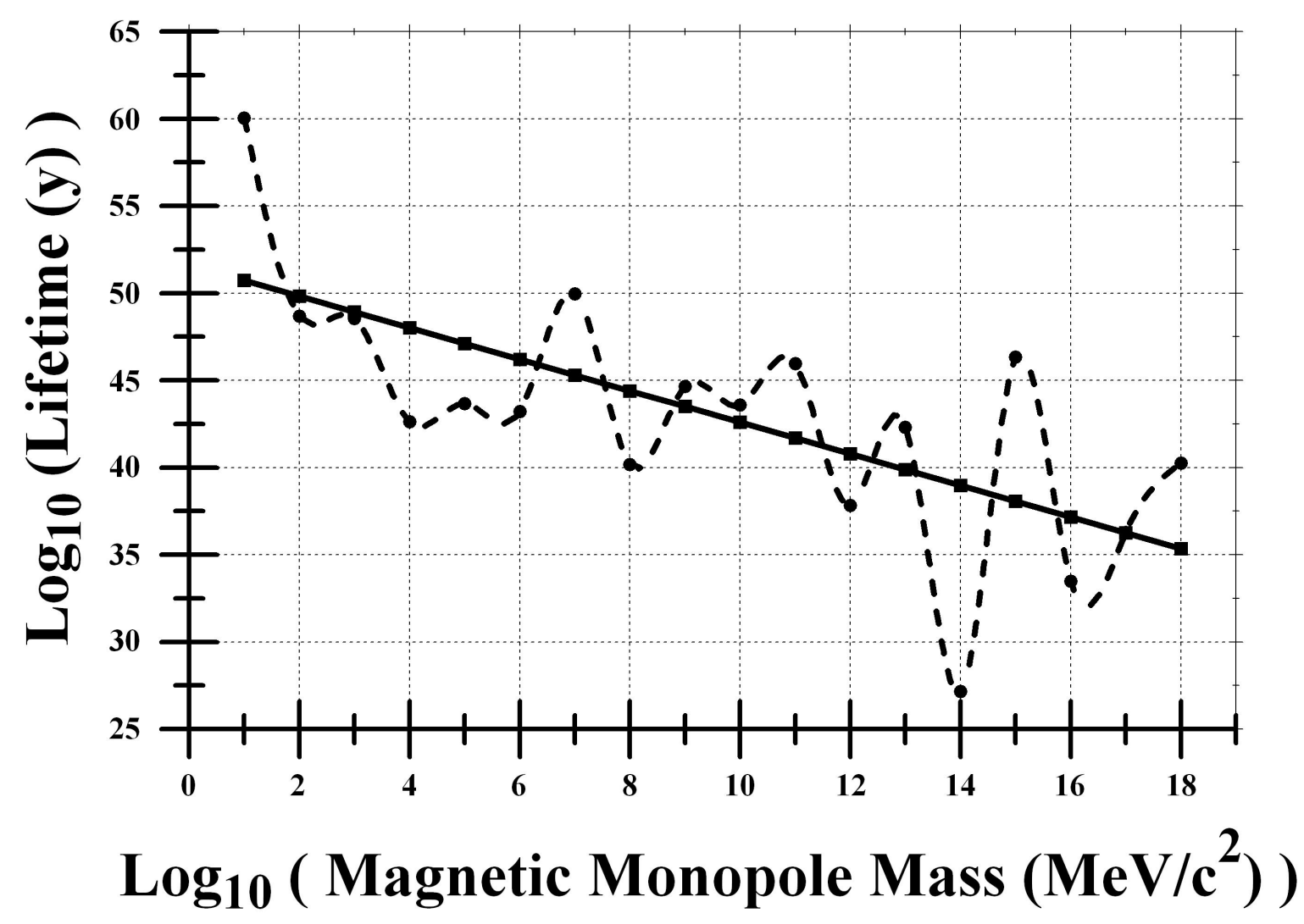

Figure 5 Magnetic monopole mean lifetime as a function of magnetic monopole mass

Magnetic monopole lifetime values derived from Eq. 10 decrease from about $10^{50}$ to $10^{35} \mathrm{y}$ for the range of mass values between 10 and $10^{18} \mathrm{y}$, respectively. These lifetime values are smaller than the predicted neutrino lifetime values ${ }^{29}$, but similar to the proton and electron values ${ }^{28}$.

The predicted magnetic monopole lifetime range is similar to that for the string model lifetimes for the proton and electron [28]. Nonrelativistic string model predictions for the proton (electron) lifetime are $10^{37}$ $-10^{58}$ y $\left(10^{29}-10^{59} \mathrm{y}\right)$, respectively. The relative consistency of the string density, length, and amplitude values for the proton, electron, and neutrino further support a long-lived value for the magnetic monopole lifetime 28,29 .

\subsection{Generalization to Closed String Models}

Bagchi et al. ${ }^{26}$ note that there is a natural emergence of an open string from a closed string given selected parameter limits. There is also a condensation of perturbative closed string modes to an open string. Reference 26 provides an important calculation that has the potential to generalize the open string model of this paper to closed string models.

\subsection{Conclusions}


The proposed nonrelativistic open string model with fixed endpoints provides an initial set of magnetic monopole string parameters that yield mean lifetime values that decrease from about $10^{50}$ to $10^{35} \mathrm{y}$ for the range of mass values between 10 and $10^{18} \mathrm{Mev} / \mathrm{c}^{2}$, respectively. The derived monopole string parameters and lifetime values are based on a simplistic open string model, and will likely change as the model becomes more complex through the inclusion of charge, electric and magnetic fields, multiple strings with loops, additional boundary conditions, and specific symmetries and gauge theories. The validity of the proposed and subsequent models will be determined by experimental verification. Experimental verification is ultimately the requirement that will determine the validity of all string theories. However, this initial set of magnetic monopole parameters provides a base case for future investigation, development, and determination of observable string characteristics.

\section{References}

[1] Polchinski, J., 2005, String Theory. Vol. 1: An Introduction to the Bosonic String, Cambridge University Press, Cambridge.

[2] Polchinski, J., 2005, String Theory. Vol. 2: Superstring Theory and Beyond, Cambridge University Press, Cambridge.

[3] Becker, K., Becker, M. and Schwarz, J. H., 2007, String Theory and M-theory: A Modern Introduction, Cambridge University Press, Cambridge.

[4] Zwiebach, B., 2009, A First Course in String Theory, $2^{\text {nd }}$ edition, Cambridge University Press, Cambridge.

[5] Green, M. B., Schwarz, J. H., and Witten, E., 2012, Superstring Theory. Vol 1: Introduction, Cambridge University Press, Cambridge.

[6] Green, M. B., Schwarz, J. H., and Witten, E., 2012, Superstring Theory. Vol 2: Loop Amplitudes, Anomalies and Phenomenology, Cambridge University Press, Cambridge.

[7] Kiritsis, E., 2019, String Theory in a Nutshell, $2^{\text {nd }}$ ed., Princeton University Press, Princeton.

[8] Vachaspati, T., Everett, A. E., and Vilenkin, A., 1984, Radiation from Vacuum Strings and Domain Wall. Phys. Rev. D30, 2046-2053.

[9] Albrecht, A., and Turok, N., 1985, Evolution of Cosmic Strings. Phys. Rev. Lett. 54(16), 1868-1871.

[10] Brandenberger, R. H., 1987, On the Decay of Cosmic String Loops. Nucl. Phys. B293, 812-828.

[11] Srednicki, M., and Theisen, S., 1987, Nongravitational Decay of Cosmic Strings. 1987, Phys. Lett. B 189(4), 397-400.

[12] Allen, B., and Shellard, E. P. S., 1990, Cosmic-String Evolution: A Numerical Simulation. Phys. Rev. Lett. 64 (2), 119-122.

[13] Bennett, D. P., and Bouchet, F. R., 1990, High-resolution Simulations of Cosmic-String Evolution. I. Network Evolution. Phys.Rev.D41, 2408-2433.

[14] Vilenkin, A. and Shellard, E. P. S., 1994, Cosmic Strings and Other Topological Defects, Cambridge University Press, Cambridge.

[15] Hindmarsh, M. B. and Kibble, T. W. B., 1995, Cosmic strings. Rep. Prog. Phys. 58, 477-562. 
[16] Olum, K. D., and Blanco-Pillado, J. J., 1999, Field Theory Simulation of Abelian-Higgs Cosmic String Cusps. Phys. Rev. D 60, 023503-1 - 023503-8.

[17] Olum, K. D., and Blanco-Pillado, J. J., 2000, Radiation from Cosmic String Standing Waves. Phys. Rev. Lett. 84, 4288-4291.

[18] Rajantie, A. K., 2003, Defect formation in the early universe. Contemp. Phys. 44, 485-502.

[19] Martins, C. J. A. P., Moore, J. N., and Shellard, E. P. S., 2004, United Model for Vortex-String Network Evolution. Phys. Rev. Lett. 92, 251601-1 - 251601-4.

[20] Davis, A.-C., and Kibble, T.W.B., 2005, Fundamental Cosmic Strings. Contemporary Physics. 46, 313322.

[21] Blanco-Pillado, J. J., Olum, K. D., and Shlaer, B., 2011, Large Parallel Cosmic String Simulations: New Results on Loop Predictions. Phys. Rev. D 83, 083514-1 - 083514-12.

[22] Lasky, P. D. et al., 2016, Gravitational-Wave Cosmology across 29 Decades in Frequency. Phys. Rev. X6, 011035-1 - 011035-11

[23] Abbott, B. et al. (LIGO Scientific and Virgo Collaborations), 2018, Constraints on Cosmic Strings Using Data from the First Advanced LIGO Observing Run. Phys. Rev. D97, 102002-1 - 102002-21.

[24] Blanco-Pillado, J. J., Olum, K. D., and Siemens, X., 2018, New Limits on Cosmic Strings from Gravitational Wave Observation. Phys. Lett. B778, 392 - 396.

[25] Particle Data Group, 2020, Progress of Theoretical and Experimental Physics. Issue 8, August 2020, 083C01. https://doi.org/10.1093/ptep/ptaa104.

[26] Bagchi, A., Banerjee, A., and Parekh, P. 2019, Tensionless Path from Closed to Open Strings. Phys. Rev. Lett.123.,111601-1 - 111601-6.

[27] Matsunami, D., Pogosian, L., Saurabh, A., and Vachaspati, T., 2019, Decay of Cosmic String Loops due to Particle Radiation. Phys. Rev. Lett. 122, 201301-1 - 201301-5.

[28] Bevelacqua, J. J. 2020, Nonrelativistic Open String Model Parameters Based on Particle Mass and Lifetime Values. Journal of Nuclear and Particle Physics 10(1), 1-8.

DOI: 10.5923/j.jnpp.20201001.01.

[29] Bevelacqua, J. J. 2020, Nonrelativistic Open String Model-Neutrino Mass and Lifetime Values, Journal of Nuclear and Particle Physics 10(2), 23 - 30. DOI: 10.5923/j.jnpp.20201002.01. 
